\title{
Utility of GeneXpert in Diagnosis of Multidrug-resistant Extrapulmonary Tuberculosis
}

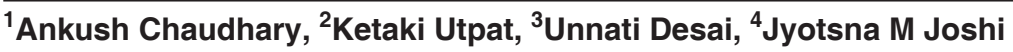

\begin{abstract}
Background: The Xpert Mycobacterium tuberculosis (MTB)/ rifampicin (RIF) assay (GeneXpert) is a rapid semi-quantitative nucleic acid amplification test with established role in the diagnosis of pulmonary tuberculosis (PTB) and multidrug-resistant (MDR) PTB. We determined the performance of the GeneXpert assay for the diagnosis of extrapulmonary tuberculosis (EPTB) MDR cases.
\end{abstract}

Aims and objectives: To study the role of GeneXpert in the diagnosis of EPTB MDR.

Materials and methods: A retrospective study was conducted over a period of 2 years at a tertiary care hospital after Ethics Committee permission. Data of 44 consecutive patients of diagnosed EPTB MDR were retrieved for GeneXpert and culture drug susceptibility test (DST). Sensitivity of GeneXpert in the diagnosis of EPTB MDR was calculated comparing culture DST results.

Results: Various EPTB MDR cases studied were lymph node TB $(n=23) 51 \%$, pleural effusion $(n=14) 32 \%$, central nervous system TB/spinal TB/psoas abscess/gluteal abscess $(n=7)$ $17 \%$. Sensitivity for GeneXpert was found to be $91.30,57$, $100 \%$ respectively. True positive and false negative were 36 and 8 cases respectively. The overall sensitivity of GeneXpert in diagnosing EPTB MDR was $81.80 \%$. The sensitivity among lymph node, pleural effusions, and spinal/psoas abscess/gluteal abscess was $91.30,57.14$, and $100 \%$ respectively.

Conclusion: GeneXpert sensitivity for the diagnosis of EPTB MDR varied with site of extrapulmonary involvement, with lower sensitivity in pleural fluid as compared with higher sensitivity among lymph node and spine TB. Nevertheless, given the rapid turnaround time and simplicity, it is a useful tool in the diagnosis of EPTB MDR when used in correct clinical context. Subsequent confirmation with culture DST, however, is recommended to diagnose false negatives.

Keywords: Drug susceptibility testing, Extrapulmonary tuberculosis, GeneXpert.

How to cite this article: Chaudhary A, Utpat K, Desai U, Joshi JM. Utility of GeneXpert in Diagnosis of Multidrugresistant Extrapulmonary Tuberculosis. Int J Recent Surg Med Sci 2017;3(2):85-87.

${ }^{1}$ Speciality Medical Officer, ${ }^{2}$ Assistant Professor, ${ }^{3}$ Associate Professor, ${ }^{4}$ Professor and Head

${ }^{1-4}$ Department of Pulmonary Medicine, Topiwala National Medical College \& B.Y.L. Nair Ch. Hospital, Mumbai, Maharashtra, India

Corresponding Author: Jyotsna M Joshi, Professor and Head Department of Pulmonary Medicine, Topiwala National Medical College \& B.Y.L. Nair Ch. Hospital, Mumbai, Maharashtra, India Phone: +912223027643, e-mail: drjoshijm@gmail.com

\section{Source of support: Nil}

\section{Conflict of interest: None}

\section{INTRODUCTION}

The emergence of drug resistance is a serious problem that poses a challenge to clinicians and hinders effective tuberculosis (TB) control across the world. ${ }^{1}$ Multidrugresistant (MDR) TB has microbial, clinical, and programmatic causes. However, it should be stressed that MDR TB is essentially a man-made phenomenon. Lack of awareness among treating physicians, improper treatment regimes, poor adherence, and drug toxicities are the prime culprits contributing to its occurrence. Treatment of MDR TB is complex due to the prolonged regimens, expensive drugs, and high incidence of drug toxicities. According to the World Health Organization $(\mathrm{WHO})^{2}$ MDR TB update 2015, about 5\% (in comparison to $3.7 \%$ in 2013) of newly diagnosed TB patients in the world have MDR TB. Lack of reliable rapid diagnostic techniques for MDR TB hampers timely diagnosis and leads to continued disease transmission, causing significant morbidity and mortality. Thus, making a rapid diagnosis of MDR TB is of utmost importance. Extrapulmonary tuberculosis MDR forms a significant proportion of the total MDR TB cases and is a major health problem in both developing and developed countries. Diagnosing drug resistance in EPTB is challenging due to its varied clinical presentations and paucibacillary nature of the disease. ${ }^{3}$ A commercially available cartridge-based nucleic acid amplification test, Xpert MTB/rifampicin (RIF) (Cepheid, USA), has been developed recently but awareness for this test among physicians is limited. GeneXpert detects the presence of TB bacilli and tests for resistance to RIF also. GeneXpert is likely to revolutionize the diagnosis and treatment of drug-resistant (DR) TB, as it is a very cost-effective and rapid test. ${ }^{4}$ Despite the recommendation by WHO since 2010 for incorporating GeneXpert as a part of diagnostic algorithm, it is till date used infrequently due to lack of awareness among physicians. There is paucity of data guiding the use of GeneXpert among EPTB MDR patients. Hence, we performed a retrospective analysis to calculate the sensitivity of GeneXpert for diagnosing EPTB MDR. Our study aimed to define the role of GeneXpert in clinical decision-making in these cases. 


\section{MATERIALS AND METHODS}

We conducted this retrospective observational study over a period of 2 years from October 2013 to October 2015 at a tertiary care center. The study was approved by Ethics Committee. Data of 44 consecutive patients of diagnosed EPTB MDR were retrieved. All patients were diagnosed cases of MDR based on GeneXpert or Mycobacterial Growth Indicator Tube (MGIT) culture DST detecting RIF resistance with clinico-radiological correlation. GeneXpert is a semi-quantitative cartridgebased nucleic acid amplification test. It is the method of choice for DST under the Revised National Tuberculosis Control Program (RNTCP). ${ }^{5}$ The MGIT culture DST was the method used for DST to confirm the results obtained by GeneXpert assay. Reports were retrieved from P. D. Hinduja Hospital, Mumbai, India. This laboratory is accredited by RNTCP for MGIT culture and first line DST. The MGIT liquid culture method is a state-of-the-art, in vitro diagnostic instrument designed and optimized for rapid detection of mycobacteria from clinical specimens. The MGIT culture DST constituted the gold standard for the diagnosis of MDR TB and for calculating the sensitivity and positive predictive value (PPV) of GeneXpert. ${ }^{5}$ These extrapulmonary cases included lymph node (cervical, axillary, abdominal, inguinal), pleural effusions, spinal TB, psoas abscess, and gluteal abscess. The demographic data, history, clinical examination findings, and investigations were noted. Corresponding GeneXpert and MGIT culture DST reports were compared and the sensitivity and PPV of GeneXpert were calculated.

\section{RESULTS}

Of the 44 patients included, lymph node, pleural effusions, and spinal/psoas abscess/gluteal abscess site of involvement was seen in 23, 14, and 7 respectively. The GeneXpert showed concordant results/confirmed MDR in $36(81.8 \%)$ of the 44 cases diagnosed on culture DST (Table 1). The true positives consisted of 21, 8 , and 7 cases of lymph node, pleural, and spinal/psoas abscess/gluteal tuberculosis. Of the 8 false-negative cases, 2 were lymph node and 6 were pleural effusion (Table 1). There were no false negatives in spinal TB/psoas abscess/gluteal abscess patients (Table 1). The sensitivity and PPV of GeneXpert in diagnosing EPTB MDR were calculated. The overall sensitivity of GeneXpert in the diagnosis of EPTB MDR was $81.80 \%$ and PPV was $100 \%$ (Table 1) comparing with culture DST for RIF resistance. Comparison with extended DST for second-line drugs was not done as extended DST was not available under the program. We observed that GeneXpert sensitivity varied according to the site of EPTB. Sensitivity among lymph node, pleural effusions, and spinal/psoas abscess/gluteal abscess was 91.30, 57.14, and $100 \%$ respectively.
Table 1: Performance characteristics of Xpert MTB/RIF in the diagnosis of extrapulmonary MDR TB

\begin{tabular}{|c|c|c|c|c|}
\hline \multirow{2}{*}{$\begin{array}{l}\text { Site/Xpert MTB } \\
\text { RIF assay }\end{array}$} & \multicolumn{2}{|c|}{$\begin{array}{c}\text { Gold standard } \\
\text { test result }\end{array}$} & \multirow[b]{2}{*}{ Sensitivity } & \multirow[b]{2}{*}{$P P V$} \\
\hline & Positive & Negative & & \\
\hline \multicolumn{5}{|l|}{ Lymph node } \\
\hline Positive & 21 & 0 & & \\
\hline Negative & 2 & 0 & $91.30 \%$ & $100 \%$ \\
\hline \multicolumn{5}{|l|}{ Pleural effusion } \\
\hline Positive & 8 & 0 & & \\
\hline Negative & 6 & 0 & $57.14 \%$ & $100 \%$ \\
\hline \multicolumn{5}{|l|}{$\begin{array}{l}\text { Spinal TB/psoas } \\
\text { abscess/gluteal } \\
\text { abscess }\end{array}$} \\
\hline Positive & 7 & 0 & & \\
\hline Negative & 0 & 0 & $100 \%$ & $100 \%$ \\
\hline \multicolumn{5}{|l|}{ Overall } \\
\hline Positive & 36 & 0 & & \\
\hline Negative & 8 & 0 & $81.80 \%$ & $100 \%$ \\
\hline
\end{tabular}

\section{DISCUSSION}

Owing to its complex and often subclinical presentations, EPTB contributes to a significant burden of mortality and morbidity, leading to a delay in diagnosis. The conventional culture DST methods are time-consuming and require trained laboratory personnel, which are a practical hurdle in resource-limited developing countries. The Xpert MTB/RIF assay (GeneXpert) is a semi-quantitative nested nucleic acid amplification test based on molecular detection of a mutated gene. It can be carried out in automated manner including bacterial lysis, nucleic acid extraction, and amplification and amplicon detection. It is cost-effective and does not require technical expertise. It enables diagnosis of DR TB within 2 hours and also has minimal false positive rates due to use of disposable closed cartridges preventing cross-contamination. ${ }^{6}$ Thus, in resource-limited settings where facilities for culture DST are not available, GeneXpert is extremely useful for rapid diagnosis of MDR TB. It was developed as a rapid test to diagnose pulmonary $\mathrm{TB}$ and to detect rifampicin resistance. ${ }^{7}$ However, it also has a significant role to play in the diagnosis of EPTB and EP MDR TB. Its potential in EPTB MDR detection has been underutilized due to lack of awareness regarding the same. Hence, we conducted the study to determine utility of this rapid and logistically simplified test in the diagnosis of EPTB MDR. We found that GeneXpert has a good overall sensitivity $(81.80 \%)$ and PPV $(97.29 \%)$ in diagnosing EPTB MDR. Hilleman et $\mathrm{al}^{8}$ found combined sensitivity of GeneXpert for EPTB to be $77.30 \%$. Significant differences in the performance of the GeneXpert test were observed in our study depending on the specimen site, higher in lymph node specimens $(91.30 \%)$, spinal/psoas abscess $(100 \%)$ but is lower in samples from pleural fluid specimens (57.14\%). Six patients 
of 14 pleural effusion patients were false negatives, which is as high as $43 \%$. This signifies the limitation of GeneXpert test in ruling out DR TB in pleural effusion cases even when the test is negative. Lower sensitivity among pleural fluid samples is cited in the literature and our findings are in concordance with the prior works. This is attributed to the physiological mechanism of formation of pleural fluid. Accumulation of pleural fluid in most cases is due to a hypersensitivity reaction to tuberculous antigens rather than direct invasion of organism into pleura. This gives a lower yield on GeneXpert. Also pleural biopsy is the sample of choice for cultures and not pleural fluid. ${ }^{9}$ Also, inhibition of polymerase chain reaction by inhibitors in pleural fluid and also by the presence of blood in fluid is also a possible explanation. ${ }^{10}$ According to a metaanalysis findings of Denkinger et $\mathrm{al}^{11}{ }^{11}$ sensitivity among pleural fluid was $46 \%$ and sensitivity among lymph node specimens was $83 \%$. Another meta-analysis finding of Penz et $\mathrm{al}^{12}$ suggested sensitivity among pleural fluid was $37 \%$ and lymph node specimen was $87 \%$. A recently published study by Sharma et $\mathrm{al}^{13}$ regarding the utility of GeneXpert in diagnosing EPTB has shown an overall sensitivity of $71 \%$ and PPV ranging from 98 to $100 \%$. But direct comparisons could not be drawn with our study as these prior works included both drug-sensitive and DR cases. In this study, we observed modest sensitivity of GeneXpert in the diagnosis of EPTB MDR. GeneXpert sensitivity varied with site of extrapulmonary involvement, with lower sensitivity among specimens obtained from pleural fluid and higher sensitivity among lymph node specimens, which is consistent with other studies in the literature. ${ }^{11-13}$

The prevalence of EPTB MDR is showing a rising trend. The diagnosis is challenging due to heterogeneous clinical presentations, paucibacillary nature, and difficulties in specimen procuring often required invasive tests. This calls for an urgent need for a rapid sensitive, technically simplified, and cost-effective tool for its diagnosis. This can aid in early initiation of appropriate therapy, leading to improved treatment outcomes, thereby minimizing the morbidity and mortality. Our findings suggest that GeneXpert plays a pivotal role in EPTB MDR diagnosis, particularly in high-burden and resource-limited settings. Awareness among physicians regarding the diagnostic utility of GeneXpert in EPTB MDR should be increased. Hence, we conclude that GeneXpert should be routinely used for rapid diagnosis even in EPTB MDR while exercising precaution in pleural effusion cases. Limitation of our study was limited number of cases as prevalence of EPTB MDR is low. Also we could not calculate specificity since we included cases diagnosed only based on the GeneXpert positivity with subsequent comparison with culture reports. Nevertheless, we conclude that GeneXpert has high sensitivity, which coupled with its speed and simplicity make it a useful tool in the diagnosis of EPTB MDR when used in correct clinical context. This also calls for future studies involving larger sample sizes for a better understanding and practical application.

\section{REFERENCES}

1. Sharma SK, Mohan A. Multidrug-resistant tuberculosis: a menace that threatens to destabilize tuberculosis control. Chest 2006 Jul;130(1):261-272.

2. WHO. Multidrug-resistant tuberculosis (MDR-TB) 2016 Update. Geneva: WHO; 2016. [cited 2016 Feb 2]. Available from: www.who.int/tb/challenges/mdr/mdr_tb_factsheet. pdf.

3. Maurya AK, Kant S, Nag VL, Kushwaha RA, Dhole TN. Trends of anti-tuberculosis drug resistance pattern in new cases and previously treated cases of extrapulmonary tuberculosis cases in referral hospitals in northern India. J Postgrad Med 2012 Jul-Sep;58(3):185-189.

4. Hakeem A, Hussain MS, Sarwar MI. Gene Xpert MTB/RIF - a novel diagnostic tool for tuberculosis in pulmonary samples. IOSR-JDMS 2013 Jul-Aug;8(2):1-3.

5. Revised National Tuberculosis Control Programme Guidelines. Programmatic Management of Drug Resistant TB (PMDT) in India. New Delhi: Central TB Division, Directorate General of Health Services, Ministry of Health \& Family Welfare; 2012. [cited 2016 Mar 9]. Available from: http://tbcindia.gov.in/WriteReadData/1892s/8320929355Guidelines\%20 for\%20PMDT\%20in\%20India\%20-\%20May\%202012.pdf.

6. Helb D, Jones M, Story E, Boehme C, Wallace E, Ho K, Kop J, Owens MR, Rodgers R, Banada P, et al. Rapid detection of Mycobacterium tuberculosis and rifampin resistance by use of on-demand, near-patient technology. J Clin Microbiol 2010 Jan;48(1):229-237.

7. Steingart K. Xpert MTB/RIF test for detection of pulmonary tuberculosis and rifampicin resistance. J Evid Based Med 2013;6:58-58.

8. Hillemann D, Rusch-Gerdes S, Boehme C, Richter E. Rapid molecular detection of extrapulmonary tuberculosis by the automated GeneXpert MTB/RIF system. J Clin Microbiol 2011 Apr;49(4):1202-1205.

9. Porcel JM. Tuberculous pleural effusion. Lung 2009 Sep-Oct;187(5):263-270.

10. Pai M, Flores LL, Hubbard A, Riley LW, Colford JM Jr. Nucleic acid amplification tests in the diagnosis of tuberculous pleuritis: a systematic review and meta-analysis. BMC Infect Dis 2004 Feb;4:6

11. Denkinger CM, Schumacher SG, Boehme CC, Dendukuri N, Pai M, Steingart KR. Xpert MTB/RIF assay for the diagnosis of extrapulmonary tuberculosis: a systematic review and meta-analysis. Eur Respir J 2014 Aug;44(2):435-446.

12. Penz E, Boffa J, Roberts DJ, Fisher D, Cooper R, Ronksley PE, James MT. Diagnostic accuracy of the Xpert MTB/RIF assay for extra-pulmonary tuberculosis: a meta-analysis. Int J Tuberc Lung Dis 2015 Mar;19(3):278-284.

13. SharmaSK, Kohli M, Chaubey J, Yadav RN, Sharma R, Singh BK, Sreenivas V, Sharma A, Bhatia R, Jain D, et al. Evaluation of Xpert MTB/RIF assay performance in diagnosing extrapulmonary tuberculosis among adults in a tertiary care centre in India. Eur Respir J 2014 Oct;44(4):1090-1093. 